Zealanders, I believe that another virus was responsible for the majority of the British cases of benign lymphocytic meningitis. As far as we could ascertain at the time this was not the virus of choriomeningitis.

It would be of great interest to us here if Dr. Caughey would give us his impressions of the 1947-8 outbreak of acute poliomyelitis in New Zealand, especially as to the incidence of unusual types of the disease.-I am, etc.,

London, W.1.

Douglas McAlpine.

\section{Juvenile General Paralysis}

SIR,-I was very interested in the account of Dr. W. Liddell Milligan's case (May 8, p. 881), but a somewhat similar case which I encountered some years ago leads me to doubt the correctness of his conclusion, namely that a case of juvenile general paralysis had sustained a spontaneous remission.

In 1933 I was consulted by a mother regarding her son, aged 10 years 5 months, who a few months previously had begun to exhibit major epileptic attacks. In childhood, from the age of $1 \frac{3}{4}$ to $3 \frac{1}{2}$, he had suffered from "convulsions," but had been quite free from fits of any kind until he had reached his eleventh year. The patient was a well-developed, intelligent boy with no stigmata of any kind. Concomitantly with the reappearance of fits he had changed a good deal temperamentally, becoming irritable and difficult, and often refusing to go to school, etc. Purely as a "shot in the dark" I had his Wassermann reaction examined and found it +3 . (The mother's and an only younger sister's were both +2 . The father refused to come for examination). I gave the patient no anticonvulsant drugs whatever, and treatment in the main consisted of three courses of bismuth (" bismogenol") separated by roughly six-monthly intervals. From the beginning of the second course no further fits developed. A subsequent W.R. gave a negative reaction. As it was difficult enough to keep up his attendances for injections I did not dare risk examination of C.S.F. About a year ago I heard from this patient regarding another matter and he has kept fit and well.

My suggestion is that Dr. Milligan's case sustained a spontaneous remission from the symptomatic fits of congenital syphilis (fits of all kinds, organic or idiopathic, often remit in a mysterious way), but that juvenile general paralysis was a later tragic development which possibly could have been entirely prevented by correct initial treatment. Be that as it may, this interesting case fully justifies one of Dr. Milligan's main contentions-the necessity of an all-round psychiatric training for those who undertake psychological work of any kind.-I am, etc.,

Armagh, N. Ireland.

ROBERT THOMPSON.

\section{Acute Intussusception in Childhood}

SiR,--The article on acute intussusception in childhood by Drs. Brenda Morrison and Donald Court (April 24, p. 776) should be read by all surgeons and general practitioners, as it stresses the extreme importance of early diagnosis and that once the condition is suspected admission to hospital should never be delayed. My last two cases arrived over 24 hours old; both required a right hemicolectomy on account of gangrene, and fortunately they recovered.

There is one important aetiological factor which I did not see mentioned in the article, and furthermore I am unable to find any reference to it in current textbooks of surgery or in those dealing more particularly with the surgery of childhoodthat is that.in every case upon which $I$ have operated there has been present an extremely mobile caecum and ascending colon, which together with the terminal ileum were on a long mesentery. Normally, the terminal $1-2$ in. $(2.5-5 \mathrm{~cm}$.) of ileum, the caecum, and ascending colon lose their mesentery during embryonic life and become fixed to the posterior abdominal wall, but in some $15-25 \%$ of individuals the old embryological mesentery remains, leaving an extremely mobile gut. The condition, which is slightly more common in females, predisposes to distortion, displacement, or rotation of the caecum and attacks of pain in the right iliac fossa, culminating in some cases in a volvulus (v. Gardiner, R. H., British Medical Journal, 1947, 1, 83).

That intussusception is largely confined to healthy first-born male children has not been my experience, as several of my cases have been female infants. The change of feeding to a more solid form of diet at the weaning age of 6 to 9 months produces more powerful peristalsis, which fact, coupled with a mobile caecum, is in my opinion enough to initiate the intussusception. It is difficult to see how a fixed bowel can easily intussuscept.

At operation I agree that reduction should take place inside the abdominal cavity as far as possible, but it is the last " bit " which is always the most difficult to reduce. It is, however, easily delivered outside the abdomen, owing to its mobility, through a para-umbilical incision, without the trauma and shock associated with evisceration. This renders the final steps of the reduction much easier. Furthermore, if unfortunately resection and right hemicolectomy are necessary, due to gangrene, the presence of a mesentery to this part of the bowel makes mobilization an easy matter. The use of pressure enemata is an extremely dangerous procedure for two reasons : first I have known one case end fatally during the administration of the enema, and, secondly, it is impossible to tell if the intussusception has been completely reduced.

Timely diagnosis followed by careful preparation and skilled surgery are the only sure and safe measures to be employed for this serious emergency of childhood.-I am, etc.

$$
\text { Aylesbury, Bucks. }
$$

Ralph H. Gardiner.

\section{Antihistamine Drugs}

SIR,-Your leader on histamine (May 8, p. 887) comments on Southwell's negative results with "anthisan" published in the same number. You seem to be much impressed by the use of inactive control tablets, and you appear to think that this in itself guarantees a true result. But this, I submit, is not the case. The control experiment can have this result only if the drug which is tested has been given in an effective amount. For instance, if, say, 1 minim of adrenaline were given to asthmatic patients, and 1 minim of physiological saline as a control, I am sure one would obtain results similar to Southwell's : some of the patients would improve remarkably with saline, whilst some of the others would get worse. Before beginning such an experiment with dummy controls one must try to find the optimal dose of the drug in question, and this can be done only individually. Unfortunately Southwell has made no such attempt, but has given the same dosage to all patients. This dosage was rather small $(0.1-0.3 \mathrm{~g}$.), and in addition was given over long periods in three or even four daily doses. I have found that this may cause tolerance and therefore give false results. The fact that drowsiness has been noted in only 10 out of his 42 patients makes it very probable that the dosage was insufficient. In my cases (Lancet, May 1, p. 677), the optimal dosage was nearly always accompanied by drowsiness.

Another serious source of error is the assessment of results according to the patients' reports. Everybody who has tried to define with chronically asthmatic patients what they regard as " an attack" knows that their conception varies enormously. In most cases they are not sure where the chronic wheeze ends and the "attack" begins. If the condition is to be assessed over a long period the violent fluctuations which occur and which may not be influenced by any drug may render any assessment still more hazardous. There must be an additional objective measurement. The vital capacity is the only such measurement I know of, and it has proved to various authors and to myself a reliable standard. For instance, Curry (J. clin. Invest., 1946, 23, 785) has shown that by varying doses of histamine different degrees of bronchial spasm and exactly proportionate decreases of vital capacity could be induced.

It is quite evident from my own experiments (which probably were not known to you when your leader was written) that anthisan does not benefit all asthma patients. Not all of them tolerate sufficiently large doses, and in a severe attack no dose seems to be large enough. But in a moderate degree of bronchial spasm it is often effective. If a really objective method is used, for instance intravenous injection, whilst the respiration of the patients is recorded by the spirometer and by the thoracograph of Verzár, the results can be relied upon. I have recently given doses of 0.05 to $0.1 \mathrm{~g}$ in 10 experiments with 7 cases of asthma who were in a moderate asthmatic state. In 7 of the 10 experiments a striking increase (between 380 and $2,000 \mathrm{ml}$.) of the vital capacity occurred within 5 to 15 minutes. These increases could not be obtained by injections of physiological saline or glucose.

The mode of action of the antihistamine drugs, many more of which will appear in the near future, is full of problems. Their dosage varies more than that of other drugs, and the way 